\title{
Propeller shrouding influence on lift force of mini unmanned quadcopter
}

\author{
I. Penkov ${ }^{1}$ and D. Aleksandrov ${ }^{2}$ \\ ${ }^{1}$ School of Engineering, Department of Mechanical and Industrial Engineering, \\ Tallinn University of Technology, Estonia \\ E-mail: igor.penkov@ttu.ee, Phone: +3725169922 \\ ${ }^{2}$ PLM Group, Estonia
}

\begin{abstract}
This article discusses the lift force generated by a mini unmanned aerial vehicle. Shrouded propellers were considered and analyses of shroud influence on lift force and energy saving were done. By help of laboratory experiments and computational fluid dynamics simulations (propeller velocity varied from $1000 \mathrm{rpm}$ to $6000 \mathrm{rpm}$, shroud height from 20 $\mathrm{mm}$ to $60 \mathrm{~mm}$, gap from $3 \mathrm{~mm}$ to $28 \mathrm{~mm}$ ), it was shown that the shroud diameter influenced the rotor energy consumption up to $30 \%$ at velocities of more than $4000 \mathrm{rpm}$. With the increase shroud diameter (increase of distance between shroud and propeller borders), the lifting force increased. A gap of more than $30 \mathrm{~mm}$ practically did not influence the lifting force. Shroud height (from $20 \mathrm{~mm}$ to $60 \mathrm{~mm}$, gap is $28 \mathrm{~mm}$ ) also influenced the propeller efficiency (up to $10 \%$ ) on small shroud heights (up to $30 \mathrm{~mm}$ ). With the increase of the shroud height, the lift force decreased about $3 \%$ and then it increased up to $12 \%$. From a value of about 50 millimetres, this influence will be unchanged but the total lifting force will be about $5 \%$ less in comparison with the force produced by propeller without shroud.
\end{abstract}

Keywords: UAV; CFD simulation; lift force; shrouded propeller; energy saving.

\section{INTRODUCTION}

There is a wide range of applications performed by Unmanned Aerial Vehicles (UAV) in the civil sphere such as police [1], rescue [2] and fire fighter needs [3]; for example, for traffic monitoring [4], navigation [5, 6], and aerial mapping. UAVs are also used for agriculture [7-10] needs, press, television, cinematography [11, 12], marine application, pollution detection, and other fields. They have generated a great interest in industrial and academic areas [13-17] due to their small size, unique flight capacities [18-21], outstanding maneuverability and low cost. A lot of researches related to stability and controllability have been conducted [22-24]. Screw theory [25, 26] and mathematical calculation methods are also usually applied when considering mini UAVs. Application areas lead to more advanced research for increasing the level of autonomy and reducing the size of UAVs. UAVs can be classified into two main categories: fixed-wing UAVs and rotary-wing UAVs. Fixed wing UAVs [27] constitute the richest group among these categories in terms of both research and utilisation. They are able to fly for a long duration at high speeds and their design is simple in comparison with the other types of UAVs. However, these UAVs suffer from the requirement of runways or additional launch and recovery equipment for takeoff and landing. Rotary wing UAVs [28], on the other hand, are advantageous since they do not require any infrastructure for takeoff and landing and 
do not need any forward airspeed for flight and maneuvering, which make them useful particularly in urban areas and indoors. This leads to a large variety and size of rotary wing UAVs. Research and development of different UAVs are conducted in many universities around the world and also at well-known companies. A wide variety of completely manufactured UAVs or custom mini UAVs built from off the shelf parts available are using standard power sources [29] whereas energy saving is a vital issue. Lots of researchers [30] have considered this problem. One of the most important factors influencing UAV power consumption is the design of aerial vehicle. Conceptual and detailed designs need deep analyses of different parameters [31, 32]. Optimisation of size and mass parameters increase the efficiency of the flying system [33, 34] and optimisation of design increases system stability and controllability [35, 36].

The main purpose of this research is the optimisation of shrouded multicopters with respect to efficiency. Firstly, the influence of gap between propeller and shroud on lifting force produced by rotor will be considered. Then, the influence of shroud height will be analysed.

\section{METHODS AND MATERIALS}

\section{Shrouded Propellers}

The quadrotor helicopters are enclosed within a protective frame permitting flights indoors and in obstacle-dense environments with a reduced risk of damage to the vehicles, their operators or surroundings. The propellers with a protective shroud around them are usually called shrouded propellers or ducted fans. These added safety benefits greatly accelerate the design and test flight process by allowing testing to take place indoors or out, by inexperienced pilots, and with a short turnaround time for recovery from incidents [37]. The shrouds around propellers can also be used to change the path of air flow around and near the propellers. Pressure difference between upper and lower surfaces can be changed and should be verified to know the influence of shroud parameters on the lifting force [38].

\section{Theory of Shrouded Propellers}

According to the Rankine-Froude momentum theory (propeller is modelled as an infinitely thin disc, inducing a constant velocity along the axis of rotation), the thrust of a propeller ( $T$ ) at hovering flight is given by Eq. (1) [39]:

$$
T=\frac{1}{2} \rho S_{1} V_{e}^{2}
$$

where $\rho$ is air density; $S_{l}$ is the disk propeller area (Figure 1(a)); $V_{e}$ is velocity of the air stream coming out.

With a shrouded fan, direct application of the momentum theory ensures the following:

$$
T=\rho S_{e} V_{e}^{2}=\sigma \rho S_{1} V_{e}^{2}
$$

where $S_{e}$ is terminal section of the air flow (Figure 1(b)); $\sigma=S_{e} / S_{1}$ is diffusion factor. 
As shown in Eq. (1), the diffusion factor is equal to 0.5 for a free propeller and for a shrouded $\sigma$ is approximately equal to one. For special shrouds with spherical diffusors, $\sigma$ can be greater than three or five [39].

According to CFD (Computational Fluid Dynamics) optimisation results, lifting force can be increased using a shroud that has a conical geometry $\left(S_{e}>S_{1}\right)$.
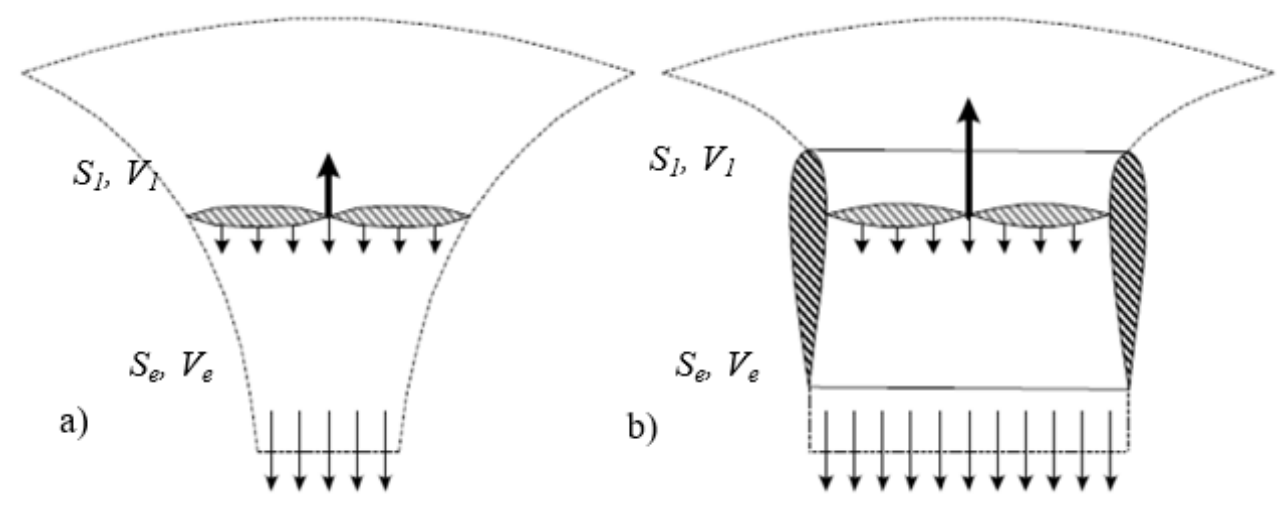

Figure 1. Airflow near the propeller without a duct (a) and with a duct (b) [39].

This research has incorporated a shroud with a shape of cylindrical tube around the propeller for safety purposes (for example, Safeflight Copters, USA). A minimal gap between the propeller and the shroud was set to $3 \mathrm{~mm}$. The use of a smaller gap (less than one millimetre) between the propeller and the shroud will work similarly to the impeller model [40] where the closeness of the internal propeller and the inner surface of the impeller housing minimises turbulences created near the rotating propeller ends. Therefore, the lifting force will improve. In usual multi-rotor helicopters, sufficiently flexible light materials (usually carbon fibre, plastic or styrofoam) are used. Creating a stiff shroud around the propeller with a gap of less than one millimetre is not reliable (mass and/or price of helicopter will increase).

\section{Simulations}

Simulations and experiments were made to understand how the shroud affected the lifting force. It is possible to modify shroud dimensions to understand how its size affects the lifting force. The diameter varied from 260 to $310 \mathrm{~mm}$ (Fig. 2) while the propeller used for calculations had a standard and not varied diameter equal to $254 \mathrm{~mm}$ (10 in). The height of the shroud changed between $20 \mathrm{~mm}$ and $60 \mathrm{~mm}$.

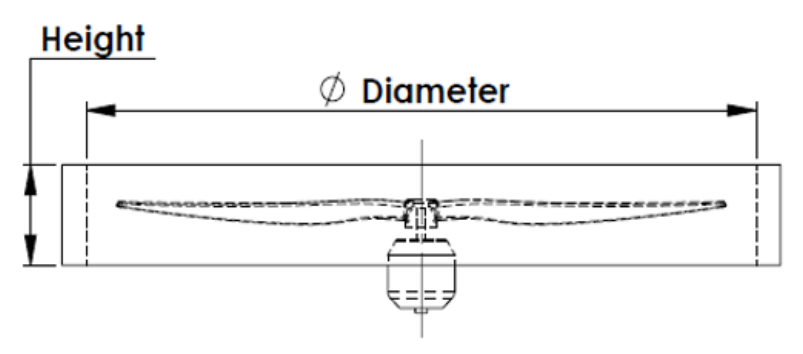

Figure 2. Changes of dimensions of the shroud around the propeller.

First, simulations were accomplished with CFD software to determine the lifting force. The lifting force produced by the propeller was determined at the propeller rotation 
speed of $5000 \mathrm{rpm}$ with a different diameter and height of the shroud. Next, the diameter of the shroud around the propeller was changed. Lifting force and motor power consumption were measured at different rotor angular velocities. Simulations and experiments for a case without a shroud around the propeller were also done. The lifting force created by propellers was calculated with the use of SolidWorks Flow Simulation. CFD results were confirmed by experiments. The propeller with diameter of $254 \mathrm{~mm}$ and standard pitch of $127 \mathrm{~mm}$ ( $10 \times 5$ inches) was used and the shroud diameter and its height were changed. All the simulations were made with rotor angular velocity of $5000 \mathrm{~min}^{-1}$.

Figure 3 shows the dependency of the lifting force produced by one propeller with a certain diameter of the shroud around it. The shroud height was fixed to $40 \mathrm{~mm}$. Minimum diameter was $260 \mathrm{~mm}$ (when the gap between the rotor and the shroud was only $3 \mathrm{~mm}$ at each side) and maximum was $310 \mathrm{~mm}$. The graph shows that with an increase in the shroud diameter, the lifting force also increased. This happened because of airflow going into the upper part of the system and did not cover the shroud (Figs. 4 and 5). The dashed line shows the lifting force of the propeller without any shroud around it. It can be seen that even the gap equal to $30 \mathrm{~mm}$ decreased the lifting force but the decreasing was insignificant (about 6\%). With further increasing of gap values, the influence of shroud disappeared.

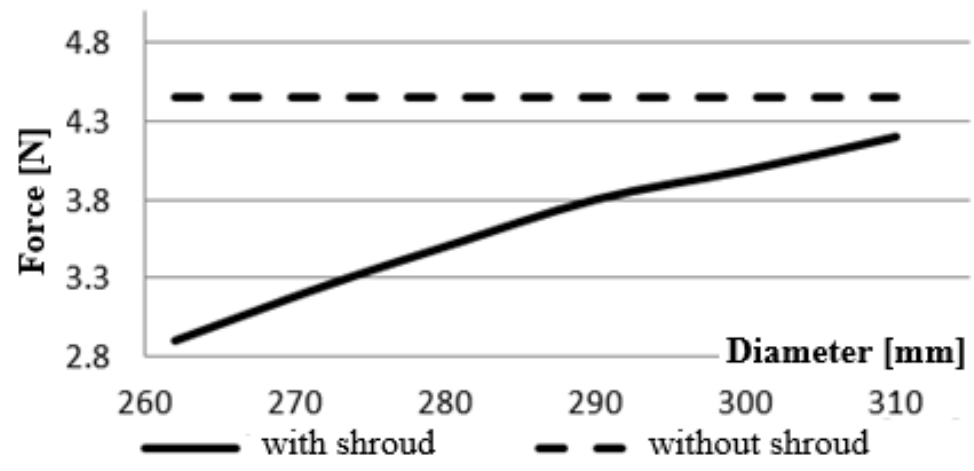

Figure 3. Experimental dependency of the lifting force on the diameter of the shroud around the propeller.

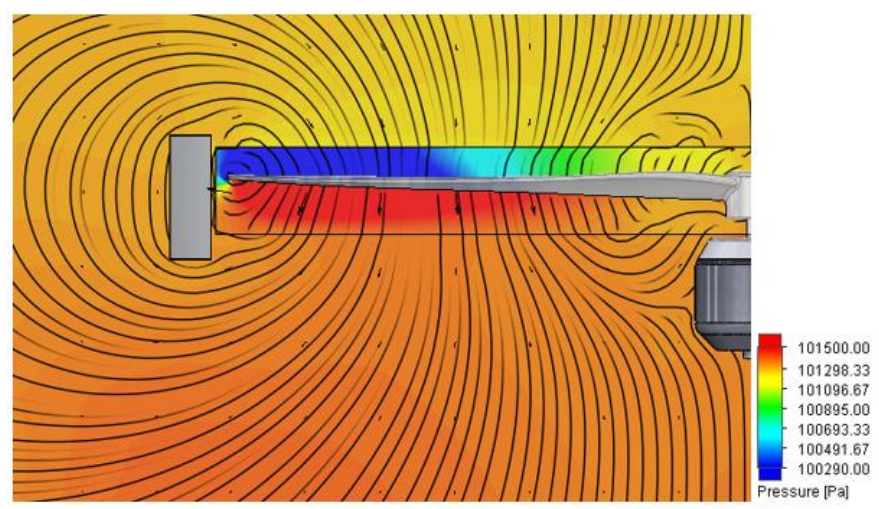

Figure 4. Pressure (colours) and velocity (vectors) distribution around the rotating propeller with the shroud with a diameter of $260 \mathrm{~mm}$, gap was $3 \mathrm{~mm}$.

Near the ends of the rotating propeller, vortices were created. Those areas led to the "overflow" of air from the higher-pressure region near the bottom surface of the 
propeller to the lower pressure area near the top propeller surface. In Figure 4, the shroud diameter was $260 \mathrm{~mm}$. When the shroud was maximally close (gap was only $3 \mathrm{~mm}$ ) to the propeller, turbulence on the propeller ends changed and the vortex went around the shroud. In Figure 4, the colours show pressure while vectors show velocity direction and flow magnitude. When the propeller was surrounded by the shroud with diameter 290 $\mathrm{mm}$ and more (gap was more than $20 \mathrm{~mm}$ ), vortex flow near the propeller ends went around the shroud partly only (Figure 5). With an increase of the shroud diameter, the propeller worked more like a separately standing (with no shroud) propeller. By changing the height of the shroud (Figure 6), the lifting force was decreasing from the height of 20 $\mathrm{mm}$ to $30 \mathrm{~mm}$. This happened because of the small shroud height and small gap. If the shroud height was very small (less than $20 \mathrm{~mm}$ ), airflow moved around it and produced a lifting force. With the increasing of shroud height (up to $30 \mathrm{~mm}$ ), airflow was divided into two parts and the inner part will produce forces in the opposite side of the lifting force. With the increasing of shroud height to $40 \mathrm{~mm}$ and more, all useful airflow will be inside of the shroud and will produce a lifting force. At a larger height (up to $60 \mathrm{~mm}$ ), the vortex moved to the inside of the formed tube (Figure 7) and this promoted a decrease of pressure difference reduction between the upper and lower surfaces of the propeller.

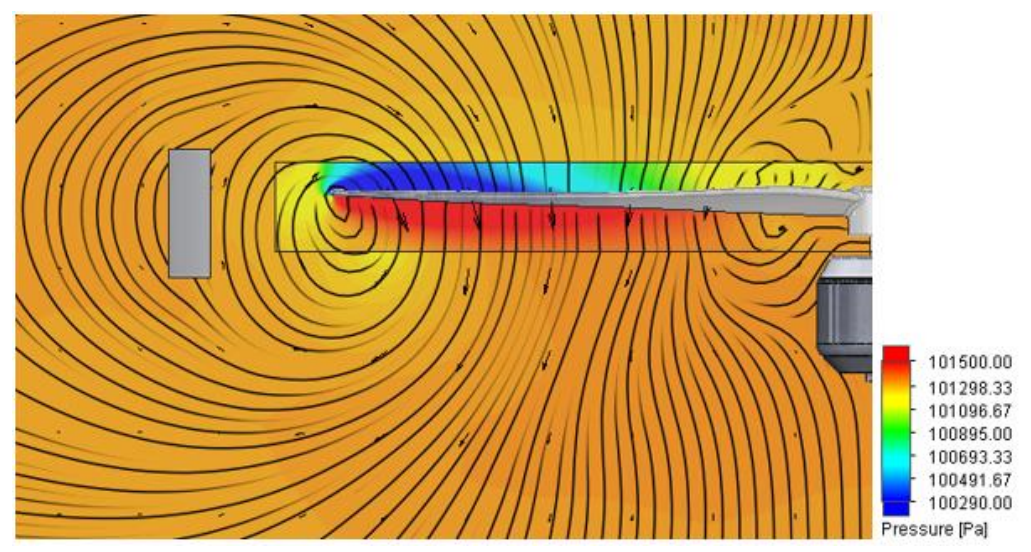

Figure 5. Pressure (colours) and velocity (vectors) distribution around the rotating propeller with a shroud diameter of $310 \mathrm{~mm}$, gap was $28 \mathrm{~mm}$.

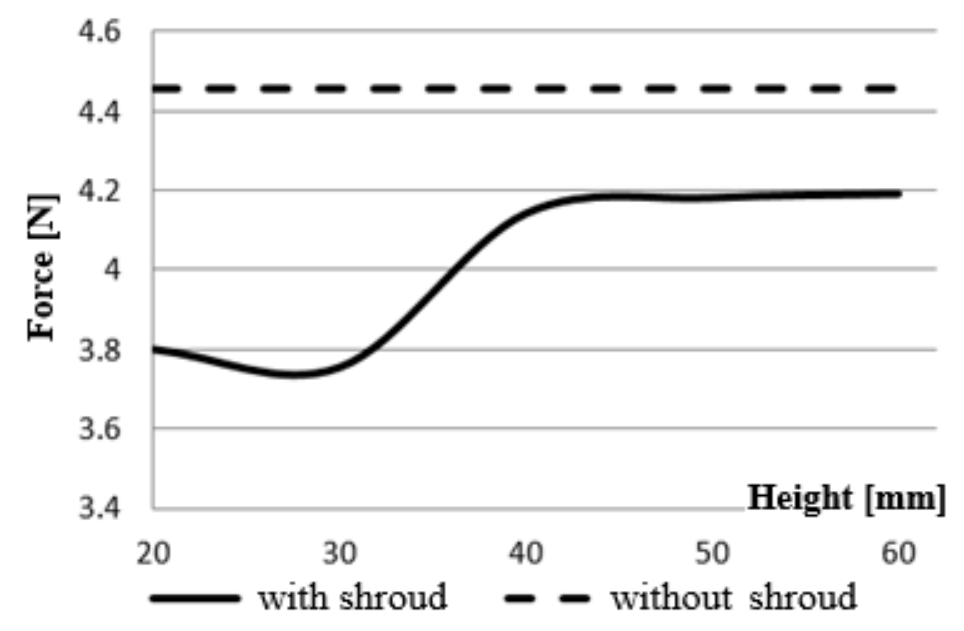

Figure 6. Dependency of the lifting force on the height of the shroud around the propeller, gap was $3 \mathrm{~mm}$. 


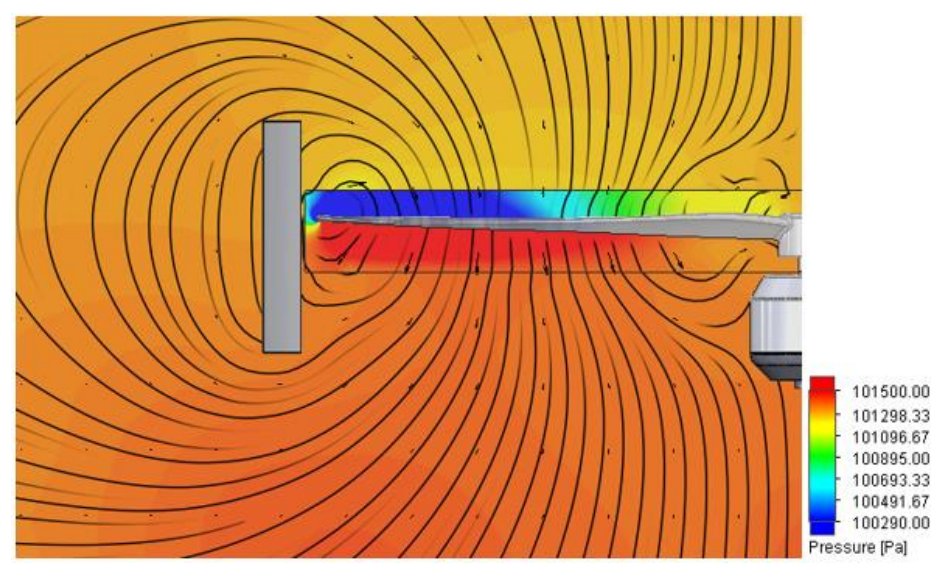

Figure 7. Pressure (colours) and velocity (vectors) distribution around the rotating propeller with a shroud height of $60 \mathrm{~mm}$, gap was $3 \mathrm{~mm}$.

\section{Experiments}

A shroud with a height of $60 \mathrm{~mm}$ and changeable diameter was made of steel sheet metal and fixed by three support stands (Figure 8). Altogether, measurements for five different diameters (from $270 \mathrm{~mm}$ to $310 \mathrm{~mm}$ with step of $10 \mathrm{~mm}$ ) of the shroud were made. Experiments without a shroud around the propeller were also done.

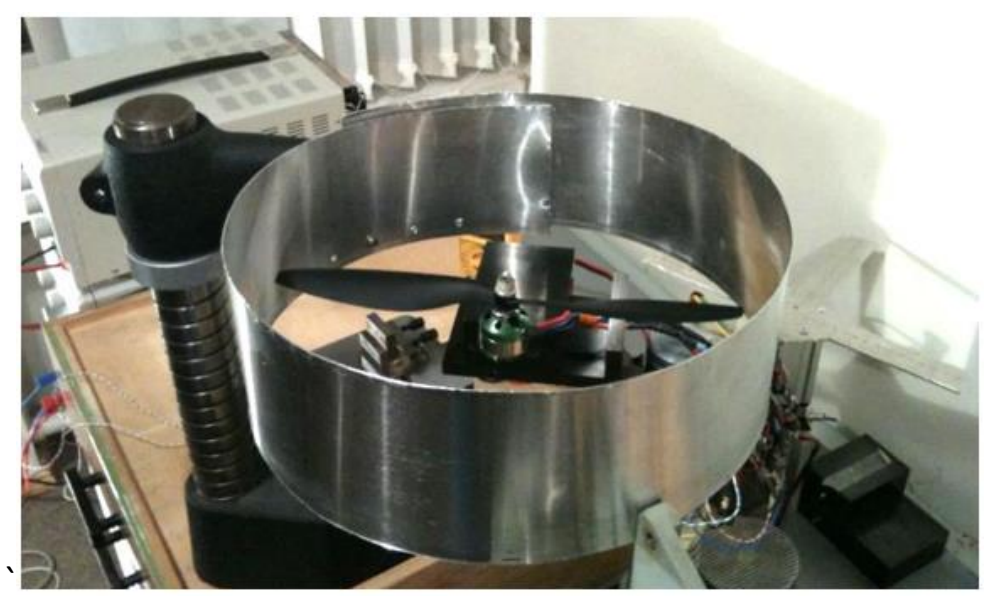

Figure 8. Experiment set up.

The graph in Figure 9 shows the dependency of the lifting force on propeller angular velocity for different diameters of the shroud around the propeller. With the increase of the shroud diameter, the lifting force produced by the propeller also increased. This happened because of airflow that moved more smoothly around the propeller edge. Magnitudes of lifting force with the increase of gap values approached the lifting force produced by the propeller without any shrouds. Graphs in Figure 9 and 10 have a great correlation where the experiments were made at the propeller angular velocity of 5000 $\min ^{-1}$. During the experiments, power consumption measurements were also made using a bypass resistor. Figure 11 shows the dependency of the lifting force on the motor's power consumption. With the increase of the shroud diameter, the rotating propeller produced a higher lifting force and the system efficiency increased (power consumption decreased). 


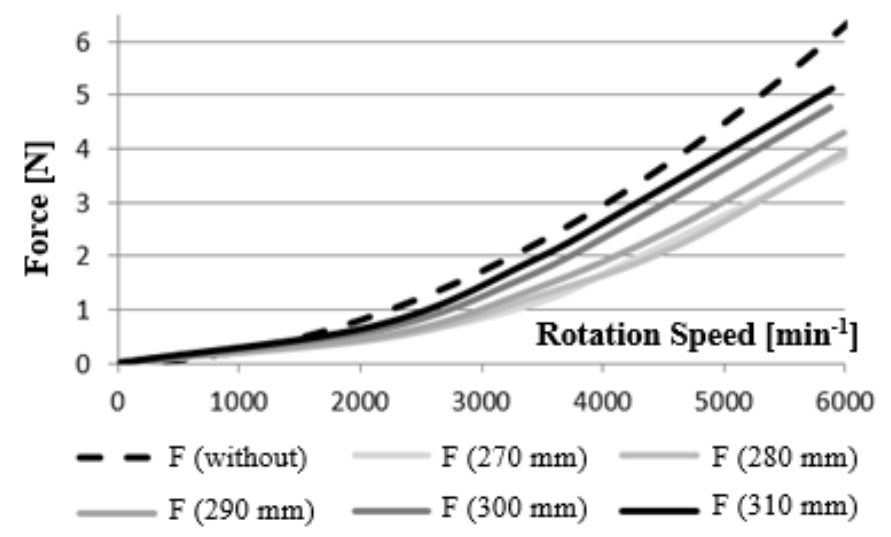

Figure 9. Dependency experiments of the lifting force on the propeller rotation speed for different diameters of the shroud around the propeller.

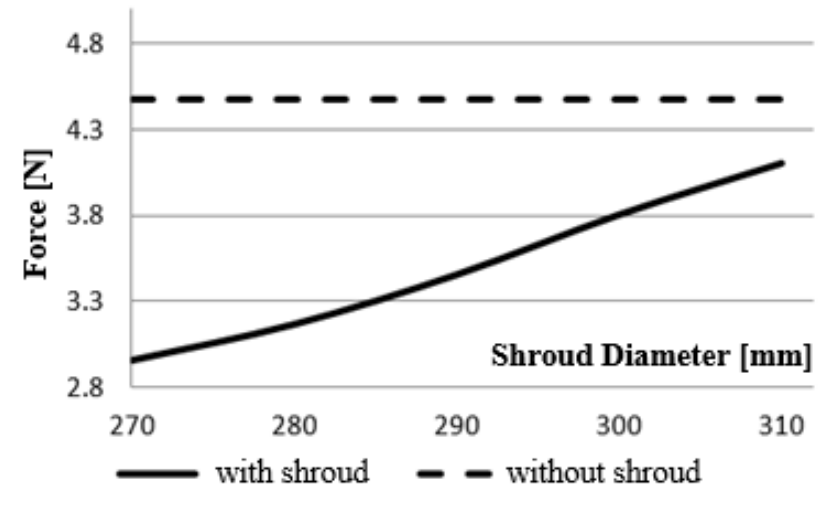

Figure 10. Dependency experiments of the lifting force on the diameters of the shroud around the propeller.

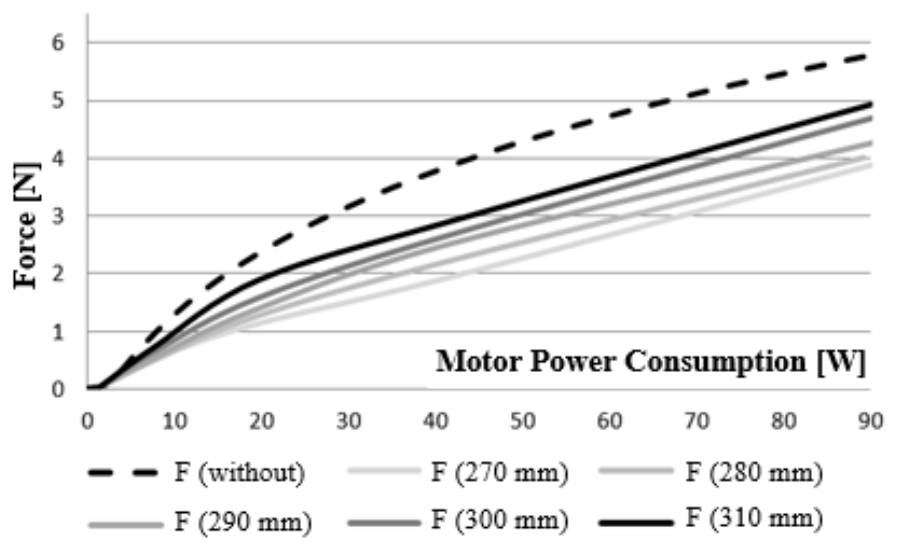

Figure 11. Dependency experiments of the lifting force on the motor power consumption.

The propeller created the highest lifting force when the shroud around the propeller was not used. Changing of the shroud diameter led to changing the lifting force. The smaller shroud diameter leads to the smaller the lifting force that can be produced at certain angular velocity and certain motor power. With an increase in the shroud height, 
the lifting force increased up to $40 \mathrm{~mm}$ in height and then stabilised (similar to the rotor working in a long tube). The lifting force of a propeller with different diameters and height dimensions of straight safety shroud around it was less than the force produced by the propeller without the shroud.

\section{CONCLUSIONS}

The lifting force depends on the height of the shroud around the propeller and distance between the propeller and shroud. By changing the height of the shroud (with gap equal to $3 \mathrm{~mm}$ ), the lifting force decreased about $3 \%$ from the height of $20 \mathrm{~mm}$ to $30 \mathrm{~mm}$ and then increased about $12 \%$ with the increase of shroud height to $50 \mathrm{~mm}$. From a shroud height of $60 \mathrm{~mm}$, the magnitude of lifting force stabilised and it was about $5 \%$ less than the force produced by the propeller without shroud. Shroud diameter (gap) also influenced the lifting force. At small gaps $(3 \mathrm{~mm})$, the lifting force decreased about $30 \%$ in comparison to the propeller without shroud. With the increase of the gap, lifting force increased but even at large gaps (more than $30 \mathrm{~mm}$ ), it will be about $6 \%$ less than the force produced by the propeller without shroud.

\section{ACKNOWLEDGEMENTS}

This research work was supported by innovative Manufacturing Engineering Systems Competence Centre IMECC (supported by Enterprise Estonia and co-financed by the European Union Regional Development Fund, project EU48685); Estonian Research Council grant PUT1300; the Estonian Centre of Excellence in Zero Energy and Resource Efficient Smart Buildings and Districts, ZEBE, grant TK146 funded by the European Regional Development Fund.

\section{REFERENCES}

[1] Coifman B, McCord M, Mishalani RG, Iswalt M, Ji Y. Roadway traffic monitoring from an unmanned aerial vehicle. IEE Proceedings-Intelligent Transport Systems; 2006. p. 11-20.

[2] Ryan A, Hedrick JK. A mode-switching path planner for UAV-assisted search and rescue. 44th IEEE Conference on Decision and Control and European Control Conference; 2005. p. 1471-6.

[3] Casbeer DW, Kingston DB, Beard RW, McLain TW. Cooperative forest fire surveillance using a team of small unmanned air vehicles. International Journal of Systems Science. 2006;37:351-60.

[4] Xiaofeng L, Zhongren P, Zhang L, Li L. Unmanned aerial vehicle route planning for traffic information collection. Journal of Transportation Systems Engineering and Information Technology. 2012;12:91-7.

[5] Courbon J, Mezouar Y, Guénard N, Martinet P. Vision-based navigation of unmanned aerial vehicles. Control Engineering Practice. 2010;18:789-99.

[6] Mustafah YM, Azman AW, Akbar F. Indoor uav positioning using stereo vision sensor. Procedia Engineering. 2012;41:575-9.

[7] Xiang H, Tian L. Development of a low-cost agricultural remote sensing system based on an autonomous unmanned aerial vehicle. Biosystems Engineering. 2011;108:174-90. 
[8] Roldán JJ, Joossen G, Sanz D, del Cerro J, Barrientos A. Mini-UAV based sensory system for measuring environmental variables in greenhouses. Sensors. 2015;15:3334-50.

[9] Roldán JJ, Sanz D, del Cerro J, Barrientos A. Lift failure detection and management system for quadrotors. ROBOT2013: First Iberian Robotics Conference: Springer; 2014. p. 103-14.

[10] Roldán JJ, del Cerro J, Barrientos A. A proposal of methodology for multi-UAV mission modeling. 23th Mediterranean Conference on Control and Automation; 2015. p. 1-7.

[11] Feng L, Ben MC, Yew LK. Integration and Implementation of a Low-cost and Vision-based UAV Tracking System. Chinese IEEE on Control Conference; 2007. p. 731-6.

[12] Mejias L, Saripalli S, Campoy P, Sukhatme GS. Visual servoing of an autonomous helicopter in urban areas using feature tracking. Journal of Field Robotics. 2006;23:185-99.

[13] Poyi GT, Wu MH, Bousbaine A, Wiggins B. Validation of a quad-rotor helicopter matlab/simulink and solidworks models. 2013.

[14] Bousbaine A, Wu MH, Poyi GT. Modelling and simulation of a quad-rotor helicopter. 2012.

[15] Van Treuren KW, Hays AW, Wisniewski CF, Byerley AR. A Comparison of the Aerodynamic Performance and Aeroacoustic Behavior of Commercial and Custom Designed Quadcopter Propellers. 2017.

[16] Wisniewski CF, Byerley AR, Heiser WH, Van Treuren KW, Liller III WR. Designing Small Propellers for Optimum Efficiency and Low Noise Footprint. 33rd AIAA Applied Aerodynamics Conference; 2015. p. 2267.

[17] Wisniewski C, Byerley A, Heiser W, Van Treuren KW, Liller W. The influence of airfoil shape, tip geometry, reynolds number and chord length on small propeller performance and noise. 33rd AIAA Applied Aerodynamics Conference; 2015.

[18] Otsuka H, Nagatani K. Thrust loss saving design of overlapping rotor arrangement on small multirotor unmanned aerial vehicles. IEEE International Conference on Robotics and Automation; 2016. p. 3242-8.

[19] Otsuka H, Nagatani K. Flow visualization of separation on a shrouded rotor inlet in a crosswind. Asia-Pacific International Symposium on Aerospace Technology; 2015. p. 395.

[20] Otsuka H, Nagatani K, Yoshida K. Evaluation of Hovering Thrust Performance of Shrouded Rotors for Multi-rotor UAVs to Reduce Weight. AIAA Atmospheric Flight Mechanics Conference; 2015. p. 2015-13.

[21] Yoon J, Lee J. Approximate multi-objective optimization of a quadcopter through proportional-integral-derivative control. Transactions of the Korean Society of Mechanical Engineers A. 2015;39:673-9.

[22] Sumantri B, Uchiyama N, Sano S. Least square based sliding mode control for a quad-rotor helicopter and energy saving by chattering reduction. Mechanical Systems and Signal Processing. 2016;66:769-84.

[23] Ołdziej D, Gosiewski Z. Modelling of dynamic and control of six-rotor autonomous unmanned aerial vehicle. Solid State Phenomena: Trans Tech Publ; 2013. p. 220-5.

[24] Johnson EN, Kannan SK. Adaptive trajectory control for autonomous helicopters. Journal of Guidance Control and Dynamics. 2005;28:524-38. 
[25] E JN. Theory of Elasticity. Railways, Automobiles,. 1937:Railways, Automobiles,.

[26] Penkov I, Aleksandrov D. Axial Displacements in Ball Screw Mechanisms with Two and Four Contact-Point. International Review of Mechanical Engineering. 2011;5:1213-8.

[27] Funaki M, Hirasawa N. Outline of a small unmanned aerial vehicle (Ant-Plane) designed for Antarctic research. Polar Science. 2008;2:129-42.

[28] Dittrich JS, Johnson EN. Multi-sensor navigation system for an autonomous helicopter. Digital Avionics Systems Conference, 2002 Proceedings The 21st: IEEE; 2002. p. 8C1-8C1.

[29] Longest flight for small UAV using Protonex fuel cell. Fuel Cells Bulletin. 2008;2008:5.

[30] Amoiralis EI, Tsili MA, Spathopoulos V, Hatziefremidis A. Energy efficiency optimization in uavs: a review. Materials Science Forum: Trans Tech Publications; 2014. p. 281-6.

[31] Hassanalian M, Khaki H, Khosravi M. A new method for design of fixed wing micro air vehicle. Proceedings of the Institution of Mechanical Engineers, Part G: Journal of Aerospace Engineering. 2015;229:837-50.

[32] Hassanalian M, Abdelkefi A. Design, manufacturing, and flight testing of a fixed wing micro air vehicle with Zimmerman planform. Meccanica. 2017;52:1265-82.

[33] Gertler J. US unmanned aerial systems. Library of Congress Washington Dc Congressional Research Service; 2012.

[34] Serokhvostov S. Ways and technologies required for MAV miniaturization. Proceedings of the European Micro Air Vehicle Conference; 2008.

[35] Kendoul F. Survey of advances in guidance, navigation, and control of unmanned rotorcraft systems. Journal of Field Robotics. 2012;29:315-78.

[36] Valavanis KP. Advances in unmanned aerial vehicles: state of the art and the road to autonomy: Springer Science \& Business Media; 2008.

[37] Hoffmann GM, Huang H, Waslander SL, Tomlin CJ. Quadrotor helicopter flight dynamics and control: Theory and experiment. Proc of the AIAA Guidance, Navigation, and Control Conference; 2007. p. 4.

[38] Gebauer J, Kočí P, Šofer P. Multicopter potentialities. 13th International on Carpathian Control Conference; 2012. p. 194-7.

[39] Zhang W, Fan N, Wang Z, Wu Y. Modeling and aerodynamic analysis of a ducted-fan micro aerial vehicle. Proceedings of International Conference on Modelling, Identification \& Control; 2012. p. 768-73.

[40] Gebauer J, Kočí P. Measurements of Features of the Ducted Fan as a Possible Actuator of a Multi-copter Vehicle. 12th International Conference on Carpathian Control; 2011. p. 127-30. 